\title{
La Transculturalità della Compagnia delle PoETE: Un FEnomeno InNovativo nelL’'Ambito Della LetTeratura Italiana della Migrazione Mondiale ${ }^{1}$
}

\section{Begoña Pozo Ś́ncheZ*}

ABSTRACT: La Compagnia delle poete ${ }^{2}$, compagnia internazionale di scrittrici formata esclusivamente da donne che non sono nate in Italia ma che tuttavia scrivono anche in italiano, è molto innovativa nell' ambito della letteratura italiana e, oseremo dire, non solo in quella italiana, secondo le parole di Mia Lecomte, una delle fondatrici della Compagnia, raccolte da Francesco Armato. Nell'intervista a Lecomte, lei segnala che si tratta di una proposta unica poiché, fino adesso, non si hanno notizie di contributi simili in altre tradizioni culturali. Specifica anche che termini come "scrittura", "frontiera", "genere", "corpo" e "migrazione" convergono nei diversi progetti transculturali e transartistici che sono stati articolati dalla scrittura che caratterizza questa compagnia internazionale di poete italofone. I quattro spettacoli - Acromazie, Madrigne, Le altre e Novunque - che sono stati allestiti dal 2010 al 2013 congiuntamente dalle 21 poete sono il simbolo di questa precisa e innovativa scelta letteraria culturale.

PAROLE CHIAVE: Compagnia delle poete; letteratura italiana migrante e attuale; genere; frontiera.

*Universidad de Valencia

1 Questo testo si sviluppa all'interno del progetto di ricerca "La cultura como recurso de desarrollo. Prácticas, discursos y representaciones en procesos modernizadores contemporáneos" (UVEG Ministero de Educación, Cultura y Deporte).

2 D'ora in poi verrà citata come Compagnia oppure $C d p$. 
RESUMO: A Compagnia delle poete, companhia internacional de escritoras formada exclusivamente por mulheres que não nasceram na Itália, mas que escrevem também em italiano, é muito inovadora no âmbito da literatura italiana $e$, ousamos afirmar, não somente da italiana. Em entrevista a Francesco Armato, Mia Lecomte, uma das fundadoras da Compagnia, assinala também que se trata de uma proposta única, já que, até o momento, não se tem notícia de contribuições parecidas em outras tradições culturais. Especifica também que termos como "escritura", "fronteira", "gênero", "corpo" e "migração" convergem nos diversos projetos transculturais e transartísticos articulados pela escrita que caracteriza essa companhia internacional de poetas italiófilas. Os quatro espetáculos montados em conjunto pelas 21 poetas, de 2010 a 2013 - Acromazie, Madrigne, Le altre e Novunque - são o símbolo dessa precisa e inovadora escolha literária e cultural. PALAVRAS-CHAVE: Compagnia delle poete; literatura italiana migrante e atual; gênero; fronteira.

ABSTRACT: The Compagnia delle poete, an international writers' company formed exclusively by women who were not born in Italy but write also in Italian, is very innovative in the context of Italian literature and, we dare say, not only in this particular context. In the words of Mia Lecomte, one of the founders of the Compagnia, it is a unique proposition since, up to now, we have not heard of similar contributions in other cultural traditions. She also points out that terms such as "writing”, "border", "gender", "body" and "migration" converge in the various cross-cultural and trans-artistic projects articulated by the writing that characterizes this international company of poets. The four shows put together by the company's 21 poets from 2010 to 2013 - namely Acromazie, Madrigne, Le altre and Novunque - are the symbol of this accurate and innovative literary and cultural choice.

KEY WORDS: Compagnia delle poete, Italian current migrant literature; gender; border 


\section{Introduzione}

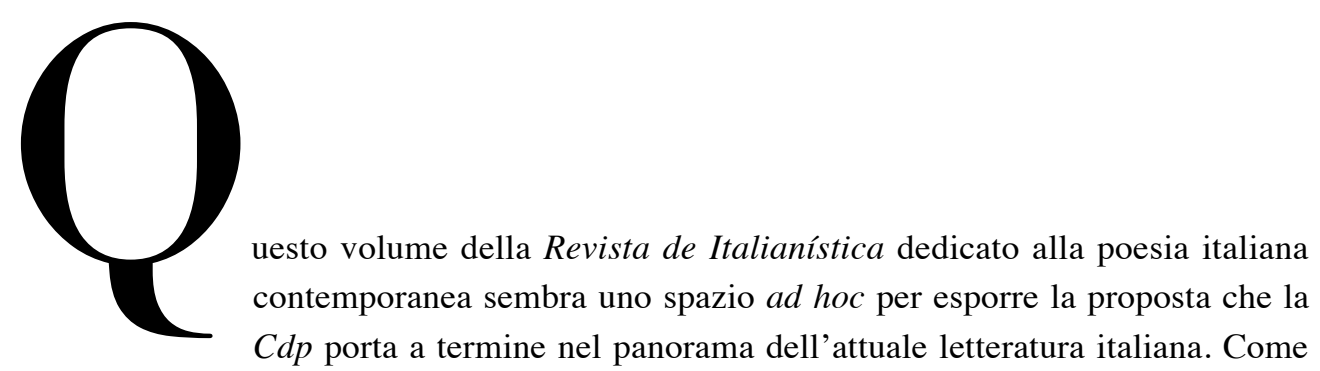

fenomeno culturale abbastanza recente, dato che la Compagnia è stata fondata nel 2009, è ancora relativamente sconosciuta tanto in Italia quanto fuori dai suoi confini, anche se in Italia pian piano il suo lavoro e la sua proposta transculturale stanno ottenendo un certo eco, per cui questo volume risulta la cornice ideale in cui poter presentare e parlare del loro lavoro oltre le frontiere del paese transalpino.

Per spiegare con quattro parole il fenomeno prima di specificarlo in modo più particolareggiato, diremo che si tratta di una compagnia internazionale di poete formata esclusivamente da donne 
che non sono nate in Italia ma che tuttavia scrivono anche o soprattutto in italiano. Questa caratteristica è totalmente innovativa nell' ambito della letteratura italiana migrante mondiale e oseremo dire non solo in quella italiana, seguendo le parole di Mia Lecomte, poeta fondatrice della Compagnia, raccolte da Francesco Armato nel suo recente volume Premiata Compagnia delle Poete (2013). Nell'intervista a Lecomte che si trova nelle pagine finali del libro, la scrittrice segnala che si tratta di una proposta unica poiché, fino adesso, non si hanno notizie di contributi simili in altre tradizioni culturali. Specifica anche che termini come "scrittura", "frontiera", "genere", "corpo" e "migrazione" convergono nei diversi progetti transculturali e transartistici che sono stati articolati dalla scrittura che caratterizza questa compagnia internazionale di poete italofone. I quattro spettacoli - Acromazie, Madrigne, Le altre e Novunque - che sono stati allestiti dal 2010 al 2013 congiuntamente dalle 21 poete sono la prova di questa precisa e innovativa scelta letteraria e culturale.

\section{La proposta transculturale della $C d p$}

Dopo queste indicazioni generali è il caso, sempre limitandoci allo spazio di questa esposizione, di approfondire un po' di più la presentazione della Compagnia delle Poete - nella loro proposta transculturale -, per poter riflettere sul valore innovativo del loro apporto nella letteratura italiana dell'attualità.

Soffermiamoci quindi sulla prima questione, che parte proprio dal nome scelto, ossia, il definirsi come la "Compagnia delle Poete", dove l'uso del termine "poete" - e non "poetesse", come sarebbe il plurale morfologico corrispondente -emerge dal testo presente nella pagina iniziale del loro sito web. In tale scelta da loro compiuta possiamo già identificare i tratti costitutivi di questa compagnia di donne poete. Non faremo qui riferimento a tutto il frammento, perché troppo esteso, ci focalizzeremo invece solo sugli elementi essenziali per la costruzione del loro discorso e, per tanto, anche della nostra argomentazione ${ }^{3}$.

C'è quindi una serie di aspetti che vale la pena mettere in risalto partendo proprio dalle loro indicazioni che, in modo sommario, enunceremo ora a continuazione. Anzitutto si tratta di un collettivo di "poete straniere e italostraniere, almeno in parte italofone", "accomunate da una particolare storia personale di migrazione e transnazionalità" che nei loro spettacoli si basano sull'aiuto di artisti. Il loro obiettivo è di creare "una sorta di 'orchestra", utilizzando una

3 In ogni caso, come abbiamo già segnalato, si possono trovare facilmente nella presentazione che si è fatta della Compagnia nella pagina iniziale del loro sito web e che può essere consultato in diverse lingue: http://www.compagniadellepoete.com/ 
"struttura "modulare", in cui confluiscono varie tradizioni linguistiche e culturali "per riportare la poesia al pubblico, restituendola alla sua originaria funzione di oralità condivisa". I loro spettacoli, oltretutto, sono costruiti dal punto di vista "dell'ibridazione e del meticciamento" in quanto "espressione performativa e al femminile". Con tutte queste caratteristiche possiamo intuire che ci troviamo in un territorio fatto di sabbie mobili e confini sfumati e, per giunta, senz'altro conflittuali se pensiamo al loro rapporto con il concetto tradizionale di "letteratura italiana", ma affronteremo questo problema più avanti.

Ora quello che ci interessa rilevare di questa proposta è il fatto di trovarci di fronte a una compagnia "anomala" giacché la sua nascita non va letta come quella di una normale compagnia teatrale, cioè con una vocazione stabile, ma piuttosto come un'aggregazione "cangiante e fluida" - sono parole di ARMATO (2013, p. 21) - in cui nessuna delle sue componenti svolge alcun ruolo prestabilito. Assistiamo in continuazione a reinvenzioni in funzione dei bisogni che si presentano in ogni spettacolo teatrale. Da questo nasce un altro degli aspetti che caratterizzano in modo significativo questa compagnia: i suoi membri non sono delle attrici professioniste. In tutte le riflessioni, documenti, studi, ecc. apparsi finora si parla solo ed esclusivamente di "poete"; perciò abbiamo una compagnia che propone spettacoli teatrali ma non è una compagnia nel senso tradizionale né è formata da attrici. Allora, che cos'è? È qui che dobbiamo girare pagina rispetto all'associazione più evidente, cioè, quella del "corpo di attori o ballerini formato per le rappresentazioni in un teatro" (DRAE, traduzione nostra) ${ }^{4}$, per ricorrere a dei significati più ampi quali quelli presentati dal termine "compagnia" e che sono, secondo il DRAE: "l'effetto di accompagnare"; "persona o persone che accompagnano un altro o degli altri"; "una società o un congiunto di varie persone unite per uno stesso scopo". Ecco il significato iniziale a cui risponde l'idea di questo collettivo di donne, quello di accompagnare o di farsi accompagnare attraverso, in questo caso, la poesia - e non solo attraverso la poesia, come vedremo più avanti. D'altra parte, il fatto che le sue componenti non si dedichino professionalmente al mondo del teatro non toglie in alcun caso la volontà di creare spettacoli poliartistici per contribuire ad accostare la parola poetica a un pubblico. In quest'occasione, le loro azioni rispondono perfettamente alla seconda definizione del dizionario della DRAE, in cui si definisce il teatro come un posto o luogo in cui si realizza un'azione di fronte a degli spettatori o dei partecipanti, per cui, anche se a priori possa sembrare tutto il contrario, ci si basa in realtà sugli elementi minimi e necessari - testi, poete, attrici, scenario e pubblico - per realizzare "azioni" teatrali attraverso una "compagnia", anche se si tratta di una compagnia "di poete".

4 "Cuerpo de actores o bailarines formado para representar en un teatro" (Diccionario de la Real Academia de la Lengua Española). 
Dopo averne evidenziato gli aspetti conformativi, passiamo ora a segnalare gli elementi determinanti per il rafforzamento dell'altro grande pilastro che regge la Compagnia: la creazione di spettacoli a partire da testi poetici. Dal debutto nel 2010 con Acromazie, quest'associazione di poete ha scelto la forma teatrale come piattaforma attraverso la quale è possibile non solo garantire gli incontri collettivi con il pubblico ma anche incoraggiarli tramite contatti diretti. La volontà è quella - soprattutto considerando che nel mercato editoriale la poesia comprende un numero tendenzialmente ridotto di lettori e lettrici -, di accorciare le distanze tra chi sta sul palcoscenico e chi sta in platea, ottenendo in questo modo un altro dei loro obiettivi prioritari: "tradurre il mondo in italiano e trasformare l'orizzonte artistico e culturale in una prospettiva mondializzante per tutti, poete e poeti migranti e cittadini italiani della poesia" (ARMATO, 2013 , p. 21). In questo senso, come segnala il critico italiano, bisognerebbe anche chiedersi qual è il significato di un'operazione teatrale "temeraria e impura" come quella della $C d p$ nell'Italia del 2014. È evidente inoltre, dopo quattro spettacoli, che la realizzazione degli spettacoli stessi si è trasformata in un segno d'identità della $C d p$ cui, per il momento, non sembrano disposte a rinunciare. In realtà, l'organicità delle loro proposte si basa su una serie di principi che sono stati mantenuti in vigore da Acromazie fino a Novunque - che è, fin ora, il loro ultimo spettacolo -, come ad esempio la costituzione del gruppo attraverso la libera associazione di donne poete - dalla nascita nel 2009 fino ad oggi il loro numero è aumentato -; il funzionamento a modo di orchestra - da una parte ogni sceneggiatura è modulare e non si basa mai sullo stesso numero di poete sulla scena; dall'altra parte la polifonia di voci ha lo scopo di riunire le diverse tradizioni linguistiche e culturali -; il recupero dell'oralità per la trasmissione delle varie poetiche e la fusione del discorso poetico con altri codici artistici.

Queste caratteristiche rispondono al paradigma progettuale che si trova alla base del nucleo ideologico della Compagnia e che potremo peraltro specificare secondo varie azioni positive che, secondo Armato, restano vincolate "all'empowerment" (2013, p. 26) quali: la solidarietà di genere, la volontà di un percorso condiviso e la fitta rete di sentimenti che unisce le donne poete con il loro pubblico. Oltretutto questi elementi sono attraversati da un profondo desiderio orizzontale, dato che ciascuna delle collaboratrici integranti, presente o assente, fa parte del coro che c'è sulla scena; per cui ciascuna delle loro voci viene ascoltata in ogni spettacolo grazie alla presenza diretta dell'autrice, o perché i testi delle autrici assenti sono recitati da quelle presenti, oppure perché vengono introdotti nello spettacolo attraverso le registrazioni. La questione è che tanto la preparazione delle sceneggiature quanto quella dello spettacolo è 
corale e democratica, rafforzando dal fondo la necessità di una comunità, di un collegamento e di un dialogo permanente - tanto sulla scena quanto fuori della scena stessa -, ponendo in primo piano la polifonia e la solidarietà artistica; caratteristiche che Armando Gnisci o Franca Sinopoli hanno definito come essenziali dello "spirito femminile" di questa compagnia, ponendo così l'accento sul suo carattere totalmente innovativo nell'ambito dell'attuale letteratura italiana.

A questo punto possiamo dire che questa proposta della $C d p$ non solo è transculturale, ma è anche profondamente umana in cui il riscatto e il recupero della formula teatrale, unita alla forza dell'oralità, risultano elementi vivificanti e salvifici delle coscienze che producono/ ricevono i testi. La presenza dei loro corpi e delle loro voci sulla scena è il modo più solido di accostare diverse realtà culturali e linguistiche agli spettatori. E quando parliamo di varie realtà linguistiche lo facciamo perché, anche se l'italiano è diventato la "lingua amante" (TOMÁS CÁMARA, 2009, p. 367), la lingua franca adoperata in tutti i montaggi, anche le altre lingue materne appaiono nelle composizioni dando luogo a un coro multilingue che apporta nuovi ritmi e musicalità, che travalica l'uso stesso dell'italiano, che allarga i suoi confini e che inquina in modo consapevole e volontario la lingua comune. E tutto ciò si realizza nella ricchezza di ciascuna delle loro manifestazioni poliartistiche dato che trasforma in flessibili le barriere della tradizione italiana (ri)umanizzandola costantemente. Con queste annotazioni avremo già in parte dato una risposta alla questione presentata previamente sul senso finale delle operazioni teatrali della $C d p$ nell' Italia del 2014. E abbiamo fornito in parte la prova del loro approccio innovativo nell'ambito della letteratura italiana della migrazione mondiale.

\section{Il passaggio dello spettacolo alle "instalaciones literarias"}

Non possiamo introdurci per ragioni di spazio nell'analisi specifica dei rapporti conflittuali della "letteratura italiana" e la soi-disant "letteratura italofona", "letteratura migrante" o "letteratura della migrazione". D'altra parte possiamo però rimandare alle riflessioni di Donata Meneghelli quando considera che "essere letteratura (e letteratura italiana) è oggi, per questi testi, una posta in gioco politica ancora prima che estética" (2011, p. 58); quando segnala che "nella contemporaneità, in ogni caso, la letteratura è il luogo di una negoziazione che è diventata strutturale, in cui le frontiere si spostano di continuo, che non ha mai fine, che ricomincia ogni volta da capo; e che ne costituisce appunto la ricchezza" (Ibid., p. 59) e quando ricorda che "la letteratura vive costitutivamente, ed è sempre vissuta della condizione migrante" (loc. cit.). 
Questo rapporto di forza tra "letteratura italiana" e "letteratura migrante" è portato al punto di massima tensione da Michele Cometa che ritiene che il concetto di letteratura nazionale debba essere sostituito da quello di "scritture che convivono in uno stesso spazio geografico" (2010, p. 100); o che l'idea di letteratura della diaspora debba essere spostata sul piano di una diaspora incessante in quanto esperienza fondatrice della letteratura. Quindi dovremo chiederci se ha o no senso l'uso di un'etichetta come "letteratura italiana della migrazione", giacché la condizione migrante è sostanza e forma specifica della letteratura stessa. Elementi come i dati personali o la biografia degli autori e delle autrici ritenuti migranti non dovrebbero essere determinanti né funzionare come un "principio di autenticità" (MENEGHELLI, 2011, p. 68), evitando così l'idea di essere scrittore in una "giurisdizione limitata" (MENEGHELLI, loc. cit.). Proprio per evitare questa situazione, Glissant rivendica il "diritto all'opacità" (1998, p. 73) dal quale partire per mettere in pratica diverse strategie di scrittura. Nel caso concreto della $C d p$ i procedimenti collettivi o la dimensione conflittuale di alcuni di essi - legati in genere all'esperienza della migrazione - non rappresentano una costante della conduzione orchestrale degli spettacoli, anche se comunque usufruiscono di spazio all'interno di questi ultimi. E questo è dovuto al fatto che i quattro montaggi realizzati fino ad oggi analizzano la situazione della costruzione culturale dei soggetti che la strutturano - ovviamente in chiave femminile -, in tal modo che il vissuto o le testimonianze personali entrino a far parte del materiale con cui si lavora per la (ri)creazione di nuove letture. Del resto non è un caso che i titoli degli spettacoli siano dei neologismi in cui, attraverso i giochi di parole e delle associazioni lessicali, si palesa l'intreccio tra i diversi territori, pubblici e privati, reali e fittizi. Forse, in questo senso, i due titoli più indicativi sono, da un lato, Madrigne, con le risonanze socio-culturali di vocaboli come "mater", "madre", "madrina" o "matrigna"; e dall'altro, Novunque, in cui spicca la fusione avverbiale di "non" e "ovunque", ossia, in tutti i posti e in nessuno allo stesso tempo - con l'insistenza quindi dello stesso paratesto sull'idea della decentralizzazione e della dislocazione caratteristiche della Compagnia e che, in qualche modo, segnano le sue mise en scene. Stando così le cose, abbiamo finora quattro spettacoli performativi in cui i testi, nella maggior parte dei casi, sono stati creati ad hoc secondo l'idea che ogni spettacolo voleva veicolare. Tutti possono essere consultationline e inoltre l'ultima proposta realizzata fino ad oggi si basa su una pubblicazione in formato libro - Novunque, Roma: Aracné, 2013 - che consente in questo modo l'accesso al materiale.

A questo punto vale la pena indicare la possibilità di un quadro teorico che raccolga le proposte della $C d p$. In questo caso, riteniamo che le riflessioni critiche di Antònia Cabanilles 
sul romanzo Hier de Nicole Brossard potrebbero perfettamente essere utilizzate per analizzare le strategie discorsive che si propongono, a partire da quelle della $C d p$ :

La instalación visual poco a poco y con muchas reticencias está empezando a ocupar un lugar en la historia del arte. El carácter efímero de esta práctica cultural, que en muchas ocasiones contraviene o ignora las leyes de las tradicionales Bellas Artes, es en parte responsable de su silenciamiento en las historias del arte y de su publicidad en los medios de comunicación. [...] me gustaría analizar cómo algunos de sus componentes definitorios (la creación de escenarios, la investigación de un material, y especialmente la actuación política, en la medida que propone nuevas formas de ver y de representar) se hallan en una práctica artística canónica: la literatura. Algunas obras literarias [...] comparten estos mismos rasgos. Dentro de la literatura son textos fronterizos que entrañan un reto creativo y político y que se sitúan en ese territorio que también designa la idea de in-between de Homi K. Bhabha. El tránsito entre lenguajes, culturas, artes o discursos es para M. R. Higonnet el ámbito preferente del estudio comparatístico. (CABANILLES, 2012, p. 89).

Come segnalato da Cabanilles, le "instalaciones visuales" sono caratterizzate dal fatto di essere effimere, di creare spazi/scenari, dalla ricerca di materiale e dall'azione politica. Sono tutte caratteristiche che si trovano, come Cabanilles stessa mette in chiaro, nella "altra pratica artistica canonica: la letteratura" (traduzione nostra). Tracciando un'equiparazione adeguata, indica come nell'ambito letterario possiamo parlare di testi di frontiera, di sfida creativa e politica o di passaggio tra linguaggi. E tutte queste caratteristiche sono state menzionate nelle pagine anteriori in quanto elementi costitutivi della proposta transculturale della $C d p$ basata sull'interdipendenza creativa e sulla scrittura decentrata e multilingue. Ricordiamo, a modo di sintesi, quali sono le strategie tematiche e discorsive che caratterizzano i progetti poliartistici della $C d p$ e che conseguentemente li accomunano con le cosiddette "instalaciones literarias":

- Il multilinguismo: con l'uso centrale dell'italiano - se pure in comunione con altre lingue.

- La multidisciplinarità artistica: con il ruolo fondamentale della letteratura - le discipline di spicco sono la poesia e il teatro -, seppure in tutti gli spettacoli si faccia ricorso alla musica, la danza, i montaggi visivi, la fotografia e la pittura. 
- Il preminente carattere autobiografico: legato all'idea di testimonianza come "valore" proprio della letteratura migrante, per cui i testi compiono il passaggio da un "autobiografismo di finzione" ad una "autobiografia del dolore" (SINOPOLI), ossia, nella quale viene messa in scena la difficoltà di mantenere l'equilibrio tra realtà, mondo e spazio che vengono rappresentati come territori che si incrociano e attraversano, in contatto e in conflitto permanente.

- Il ricorso a una scrittura decentrata che è: mobile - gli adattamenti della sceneggiatura e del copione dipenderanno da ogni spettacolo -, flessibile - perché varierà secondo i testi da creare e dagli spazi in cui saranno rappresentati - e policentrica - perché diretta in forma collettiva e in funzione dei temi o dei testi che daranno luogo alla sceneggiatura finale.

\section{Epilogo}

Per concludere, come se gli spettacoli fossero pagine del "Libro di arena" di Borges, nessuna delle loro funzioni sarà identica, ciascuna sarà unica e irripetibile, a partire dal fatto stesso della creazione solidale e collettiva in cui il valore della creazione individuale si sgretola a vantaggio della visione comune che si vuole trasmettere allo spettatore. E proprio in questa fessura in cui convivono poesia, scena e silenzio si colloca il loro instabile "stare nel mezzo": proprio lì, in questo vuoto, è il posto in cui si può (ri)creare la costruzione complessa dell'identità ed è lì dove ancora resta spazio per poter respirare.

\section{Riferimenti bibliografici}

ARMATO, F. Premiata Compagnia delle Poete. Roma: Cosmo Iannone, 2013.

CABANILLES, A. Instalaciones literarias. In: FERRER, R. A.; RICO F. C. (Eds.). Literatura y espectáculo. Alacant: Universitat d'Alacant \& SELGYC, 2012, p. 89-100.

COMETA, M. Studi culturali. Napoli: Guida, 2010.

DRAE - Diccionario de la lengua española (22.a ed) da Real Academia Española, 2001. http://www. rae.es/rae.html Acesso: 10/6/2015

GLISSANT, E. Poetica del diverso. Roma: Meltemi, 1998.

MENEGHELLI, D. Il diritto all'opacità. Autori, contesti, generi nella letteratura italiana della migrazione. In Scritture migranti. Rivista di scambi interculturali 5, p. 57-81, 2011.

SINOPOLI, F. "Migrazione/letteratura: due proposte di indagine critica". http://ww3.comune.fe.it/ 
vocidalsilenzio/sinopoli.htm Acesso: 22 06/2015.

TOMÁS CÁMARA, D. ¿Exilio o neoesclavismo? Identidades fragmentadas, inmigración y género en Nativas de Inongo vi-Makomè. In: MARCILLAS, I. (Ed.). Visions de l'exili: literatura, pintura i gènere . Valencia: Brosquil, 2009, p. 365-386. 